\title{
LETTER TO THE EDITOR Immune Thrombocytopenic Purpura in a 5-Month-Old Female With Rotavirus Infection
}

To the Editor: Immune thrombocytopenic purpura (ITP) in infants may occur following immunizations and usually has good response to treatment with no recurrence after booster immunization with same vaccines. ITP has not been reported to be associated with either rotavirus infection or vaccine. We would like to report the case of an infant who presented with severe gastrointestinal bleeding secondary to ITP, which developed following acute rotavirus infection after having received two doses of the oral pentavalent rotavirus vaccine.

A 5-month-old previously healthy, African American female presented to the emergency room, with low-grade fever, hematochezia, and non-bloody emesis that lasted for about $48 \mathrm{hr}$. She had received her second set of immunizations, including oral pentavalent rotavirus vaccine, 1 week prior to presentation. Physical exam revealed multiple petichiae over her entire body but no visceromegaly. Barium enema was negative for intussusception, although stool enzyme immunoassay was positive for rotavirus. Platelet count was found to be $19,000 / \mathrm{cmm}$, hemoglobin was $6.4 \mathrm{~g} / \mathrm{dl}$, and white blood cell count was $8,300 / \mathrm{cmm}$ with normal differentials. On peripheral blood smear she had normochromic, normocytic red blood cells with polychromasia and occasional schistocytes, giant platelets, and no blasts. Direct Coomb's test was negative, blood urea nitrogen, serum creatinine, coagulation studies, compliment factors, liver functions, and immunoglobulin levels were all within normal limits, lactate dehydrogenase was $399 \mathrm{U} / \mathrm{L}$; serologies for human immunodeficiency virus, Epstein-Barr virus, cytomegalovirus, and Parvovirus-B19 were negative. Her bone marrow showed normal cellularity with increased number of megakaryocytes. She was stabilized with packed red blood cell transfusion and intravenous fluids, but the platelet count dropped down to $3,000 / \mathrm{cmm}$.
Thrombotic thrombocytopenic purpura was ruled out with normal ADAMTS-13 levels, though fresh frozen plasma was given prior to this. Patient was given $1 \mathrm{~g} / \mathrm{kg}$ body weight, infusion of intravenous immunoglobulin (IVIG) in two divided doses and platelet counts rose up to $32,000 / \mathrm{cmm}$ within the next 2 days. She was discharged home with recommendations for close follow-up. She required three more doses of IVIG for recurrent drop in platelet counts, over the next 2 months, with a good response each time; while no bleeding was reported. Following the fourth dose of IVIG, her platelet count increased to $192,000 / \mathrm{cmm}$ and subsequently remained over $150,000 / \mathrm{cmm}$ without intervention. After 4 months of follow-up, she continues to do well and her hemoglobin has remained stable. She was advised not to receive the third dose of rotavirus vaccine.

The development of serious rotavirus gastroenteritis in a partially vaccinated infant is rare but is known to occur. We propose that the development of ITP in this infant might have been associated with rotavirus infection, although this could have been an incidental finding. The prevalence of rotavirus infection is very high worldwide and it would be of interest to perform an analysis examining platelet counts and hemoglobin in infants diagnosed with acute rotavirus infections.

Abdul Hafeez Siddiqui, MD*
Meera B. Chitlur, MD
Division of Hematology/Oncology
Carman and Ann Adams Department of Pediatrics
Children's Hospital of Michigan
Wayne State University School of Medicine
Detroit, Michigan

*Correspondence to: Abdul Hafeez Siddiqui, Division of Hematology/ Oncology, Carman and Ann Adams Department of Pediatrics, Children's Hospital of Michigan, Wayne State University School of Medicine, Detroit, MI. E-mail: dr_jugnoo@yahoo.com

Received 17 October 2009; Accepted 21 October 2009
(C) 2010 Wiley-Liss, Inc.

DOI 10.1002/pbc.22368

Published online 6 January 2010 in Wiley InterScience

(www.interscience.wiley.com) 